\title{
Fast Image Registration for Spacecraft Autonomous Navigation Using Natural Landmarks
}

\author{
Yun-Hua Wu $\mathbb{D},{ }^{1}$ Lin-Lin Ge $\mathbb{D}^{1},{ }^{1}$ Feng Wang $\mathbb{D},{ }^{2}$ Bing Hua $\mathbb{D}^{1},{ }^{1}$ Zhi-Ming Chen $\mathbb{D}^{1},{ }^{1}$ \\ and Feng Yu iD 1 \\ ${ }^{1}$ Micro-Satellite Research Center, Nanjing University of Aeronautics and Astronautics, Nanjing 210016, China \\ ${ }^{2}$ Research Center of Satellite Technology, Harbin Institute of Technology, Harbin 150001, China \\ Correspondence should be addressed to Yun-Hua Wu; yunhuawu@nuaa.edu.cn
}

Received 17 November 2017; Revised 27 May 2018; Accepted 8 July 2018; Published 12 August 2018

Academic Editor: Linda L. Vahala

Copyright ( 2018 Yun-Hua Wu et al. This is an open access article distributed under the Creative Commons Attribution License, which permits unrestricted use, distribution, and reproduction in any medium, provided the original work is properly cited.

\begin{abstract}
In order to satisfy the real-time requirement of spacecraft autonomous navigation using natural landmarks, a novel algorithm called CSA-SURF (chessboard segmentation algorithm and speeded up robust features) is proposed to improve the speed without loss of repeatability performance of image registration progress. It is a combination of chessboard segmentation algorithm and SURF. Here, SURF is used to extract the features from satellite images because of its scale- and rotation-invariant properties and low computational cost. CSA is based on image segmentation technology, aiming to find representative blocks, which will be allocated to different tasks to speed up the image registration progress. To illustrate the advantages of the proposed algorithm, PCA-SURF, which is the combination of principle component analysis and SURF, is also analyzed in this paper for comparison. Furthermore, random sample consensus (RANSAC) algorithm is applied to eliminate the false matches for further accuracy improvement. The simulation results show that the proposed strategy obtains good results, especially in scaling and rotation variation. Besides, CSA-SURF decreased $50 \%$ of the time in extraction and $90 \%$ of the time in matching without losing the repeatability performance by comparing with SURF algorithm. The proposed method has been demonstrated as an alternative way for image registration of spacecraft autonomous navigation using natural landmarks.
\end{abstract}

\section{Introduction}

Spacecraft autonomous navigation only needs to regularly check the spacecraft working conditions, eliminating the complex navigation computing tasks, which greatly reduces the manpower and ground facility requirements and the cost of space projects [1]. Furthermore, ground stations can be destroyed during wartime. However, with the use of an autonomous navigation system, spacecraft can still work well when the ground communication is under interruption. Among many kinds of autonomous navigation systems, autonomous navigation using natural landmarks based on machine vision is a newly proposed navigation method and has potential applications for future space missions.

For the landmark-based autonomous navigation, landmarks are used as reference and measuring objects. At first, the landmarks combined with position information are gathered and stored onboard the satellite. For on-orbit satellite, the camera can capture ground targets that have been stored in the satellite. During the period when the satellite runs over the target, several images from different view angles can be captured. These images are used as the inputs of image matching algorithm. Once the matching succeeds, the location information corresponding to this landmark is used to determine the position of the satellite. Then the orbit of the satellite is estimated by different positions. Figure 1 shows the schematic of autonomous navigation using natural landmarks.

Image registration is a vital technology for landmarkbased spacecraft autonomous navigation. However, autonomous navigation systems should be stable with real-time characteristics. In order to satisfy these stringent requirements, image registration progress must be fast and stable. This paper aims to propose an alternative method to improve the above performances. 


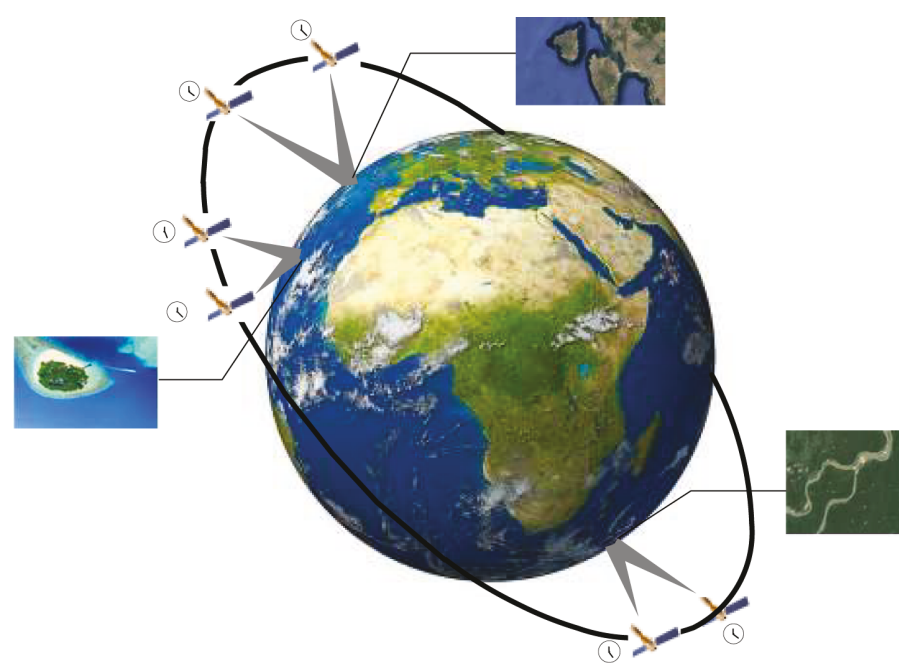

FIGURE 1: Sketch of landmark-based automatic navigation.

To improve the correctness and real-time performance in the process of image matching, Sha et al. proposed a fast matching algorithm based on image $K$-gray-degree clustering, which was robust and fast under the condition of nonlinear changing of local lighting, noise, target matching of irregular shape, and even complex background [2]. However, the problem of quickly obtaining a $K$-degree template reflecting main features of the matching object precisely still needs to be further researched. $\mathrm{Xu}$ et al. proposed a novel method called DFOB for the detection, orientation computation, and description of feature points. The method was computationally efficient as it was implemented by integral images. Compared with SIFT and SURF algorithms, the computational cost of this method was much lower [3]. However, this method does not work well under large affine and perspective deformations, making it unable to perform well in wide baseline matching.

Zhao et al. studied a method based on (principal component analysis) PCA [4] to speed up the image registration progress. The resulted computing time was reduced to $60 \%$ compared to single gray level normalized cross-correlation matching. He and Jiang proposed a fast image matching algorithm based on discrete Hartley transform (DHT) [5], which reduced data calculation and storage. Besides, it also improved image matching accuracy and efficiency. The PCAbased method proposed by Peng Zhao greatly improved the speed performance. However, its performance is poor when the rotation is larger than $20 \mathrm{deg}$. The image matching algorithm based on DHT also increased the image registration speed compared to traditional algorithm based on (fast Fourier transformation) FFT. But its computation time reached $18 \mathrm{~s}$, which cannot satisfy the real-time requirement for natural landmark-based autonomous navigation. In order to overcome the above shortcomings, a fast image registration algorithm based on chessboard segmentation is proposed. Furthermore, RANSAC algorithm is applied to remove the error-matched key points for further repeatability improvement.

The reminder of this paper is organized as follows. Firstly, Section 1 introduces the purpose of this research. Then Section 2 reviews the basic theory of PCA-SURF algorithm. Later, Section 3 presents a method called chessboard segmentation algorithm to speed up image registration progress. In addition, random sample consensus algorithm is also presented in this section to obtain the registration statistical results about the number of matches and mismatches. In Section 4 , the time consumptions of different methods are compared to verify the advantages of our algorithm. In Section 5, some metrics are defined to evaluate repeatability of image registration result. Besides, several tests are designed to evaluate the performance of the proposed method. Finally, Section 6 summarizes the contributions of this work.

\section{Review of PCA-SURF}

Principal component analysis (PCA) is a classical feature extraction and data representation technique widely used in the area of computer vision $[6,7]$. PCA-SURF, which is a combination method of PCA and SURF [8], aims to reduce the computation by compressing the data dimension.

2.1. PCA-SURF Description. Suppose that $\mathbf{U}_{i}$ is a $64 \times 1$ description vector of a reference image $I_{1}$ and $\mathbf{V}_{i}$ is a $64 \times 1$ description vector of an object image $I_{2}$. Let $\mathbf{X}$ denote all SURF description vectors in the reference image and the object image:

$$
\mathbf{X}=\left[\begin{array}{llllllll}
\mathbf{V}_{1} & \mathbf{V}_{2} & \ldots & \mathbf{V}_{n} & \mathbf{U}_{1} & \mathbf{U}_{2} & \ldots & \mathbf{U}_{m}
\end{array}\right],
$$

where $n$ and $m$ are the number of SURF features in the reference and object images, respectively. Now, a group of orthogonal projection directions is required to be found to project $\mathbf{X}$ into a lower dimension space. A covariance matrix can be defined as follows:

$$
\mathbf{R}=\mathbf{X X}^{T}
$$

Then the eigenvalue and eigenvector corresponding to $\mathbf{R}$ are $\lambda_{i}$ and $\boldsymbol{\mu}_{i}$. Actually, the value of $\lambda_{i}$ is related to the amount of information in its corresponding vector $\boldsymbol{\mu}_{i}$. Larger eigenvalue means more amount of information. Then a matrix is defined 


$$
\mathbf{C}=\left[\begin{array}{llll}
\mu_{1} & \mu_{2} & \cdots & \mu_{64}
\end{array}\right] .
$$

Here, it is important to satisfy that $\mathbf{C}$ is an orthogonal matrix. Only with orthogonal transformation, the original vectors can be invariant to their Euclidean distance or angle with each other. What is more, in order to compress the dimension with least information loss, the following relation should be satisfied:

$$
\lambda_{1}>\lambda_{2}>\cdots>\lambda_{64}
$$

Then with the orthogonal transformation, we have

$$
\mathbf{Y}=\mathbf{C}^{T} \mathbf{X}
$$

where $\mathbf{Y}$ is the feature in the new space corresponding to the original SURF feature. A new equation is defined as

$$
\mathbf{Y}^{T}=\left[\begin{array}{llll}
\mathbf{y}_{1} & \mathbf{y}_{2} & \ldots & \mathbf{y}_{64}
\end{array}\right]
$$

Here, each column $\mathbf{y}_{1} \mathbf{y}_{2} \ldots \mathbf{y}_{64}$ of $\mathbf{Y}$ is the principal component. Compressing the dimension of the descriptor, then $\mathbf{y}_{64}, \mathbf{y}_{63}, \ldots$ can be removed sequentially, because the smaller the eigenvalues are, the less the information is.

2.2. Application of Image Registration. In practical application, two conceptions called contribution rate and cumulative contribution rate should be defined:

$$
\begin{aligned}
\text { Contribution rate } & =\frac{\lambda_{i}}{\sum_{k=1}^{n} \lambda_{k}}, \\
\text { Cumulative contribution rate } & =\frac{\sum_{i=1}^{m} \lambda_{i}}{\sum_{k=1}^{m} \lambda_{k}} .
\end{aligned}
$$

Image registration task will be completed by using the first $m$ principal components which occupy a large proportion of the contribution rate. In order to find the best value of the cumulative contribution rate, some simulation results are presented in the following.

Figure 2 shows the repeatability of PCA-SURF with different cumulative contribution rates. Different lines in the figure represent the object image with different rotations. Figure 3 presents the remaining dimensions with different cumulative contribution rates. From the above comparison, it can be seen that PCA-SURF worked poorly when the rotation is larger than 20 degrees. When the rotation is less than 20 degree, the cumulative contribution rate can be chosen as 0.95 ; therefore, it can largely compress the dimension of the SURF descriptor with little accuracy loss.

\section{CSA and RANSAC}

3.1. Chessboard Segmentation Algorithm. According to [9-11], there are three strategies that can be implemented to speed up the image registration progress. The first strategy is to decrease the dimension of the key points' descriptors. And the second one is to decompose the task and deal them with multithreads. The third is to decrease the number of key points. Here, in this paper, the latter two methods are used to improve the speed performance.

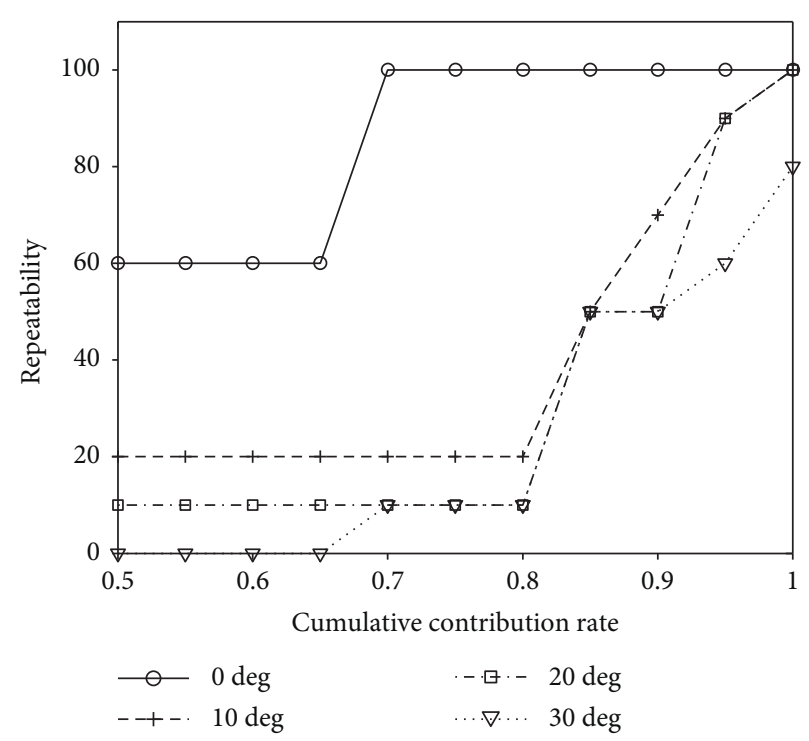

FiguRE 2: Repeatability for different cumulative contribution rates.

Chessboard segmentation algorithm, which combined these two ideas, can greatly improve the speed without losing performance of repeatability.

Figure 4 shows an island image that has been segmented into $N \times M$ parts. The number in each block represents the amount of SURF features. In order to speed up image registration progress, considering the second idea, the feature extraction tasks in each block will be allocated to different threads. Besides, by considering the third idea, only some representative blocks rather than all of them will be selected. In order to satisfy these requirements, several factors needed to be considered, for example, if the block with the most number of SURF features has been selected, then the weight of blocks around the chosen block should be decreased to make sure that regional distribution is more even. So, a block selection model should be established. The coordinate of each block is defined in Figure 4. And we suppose that $S_{i, j}$ is the amount of SURF features of block $D_{i, j}$.

Due to the diversity of each image, the SURF feature amounts for different images must be distinct so normalization is defined as

$$
\widehat{S}_{i, j}=\frac{S_{i, j}-S_{\min }}{S_{\max }-S_{\min }},
$$

where $S_{\min }$ and $S_{\max }$ are the minimum and maximum values, respectively, of all $S_{i, j}$. And $\widehat{S}_{i, j}$ is the normalization of $S_{i, j}$. It is assumed that $\widehat{S}_{i, j}$ is the weight of importance for each block. Here is the weight matrix $\mathbf{W}$

$$
\mathbf{W}=\left[\begin{array}{ccc}
\widehat{S}_{1,1} & \ldots & \widehat{S}_{1, M} \\
\vdots & \ddots & \vdots \\
\widehat{S}_{N, 1} & \ldots & \widehat{S}_{N, M}
\end{array}\right]=\left[\begin{array}{ccc}
w_{1,1} & \ldots & w_{1, M} \\
\vdots & \ddots & \vdots \\
w_{N, 1} & \ldots & w_{N, M}
\end{array}\right]
$$

Local field attenuation effect simulates the phenomenon that the importance of blocks around the selected block needs 


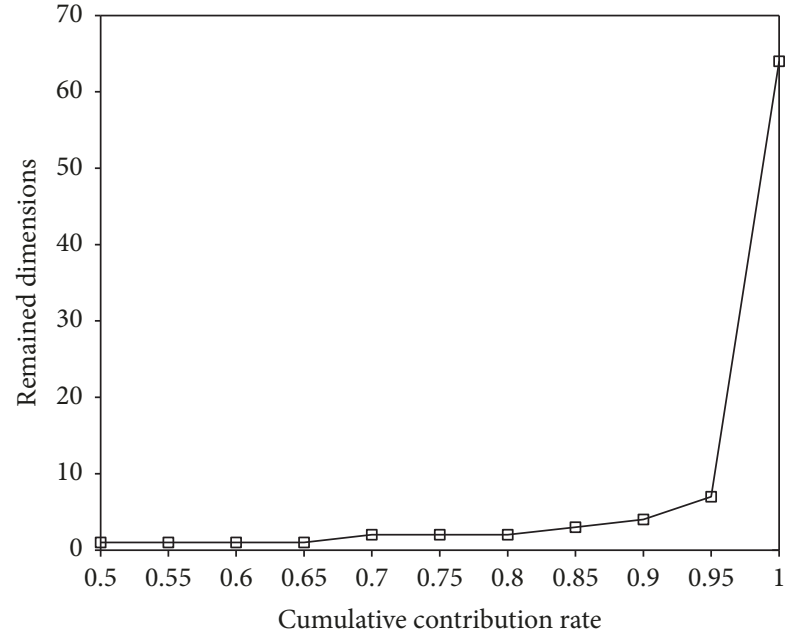

FIGURE 3: Remaining dimension for different cumulative contribution rates.

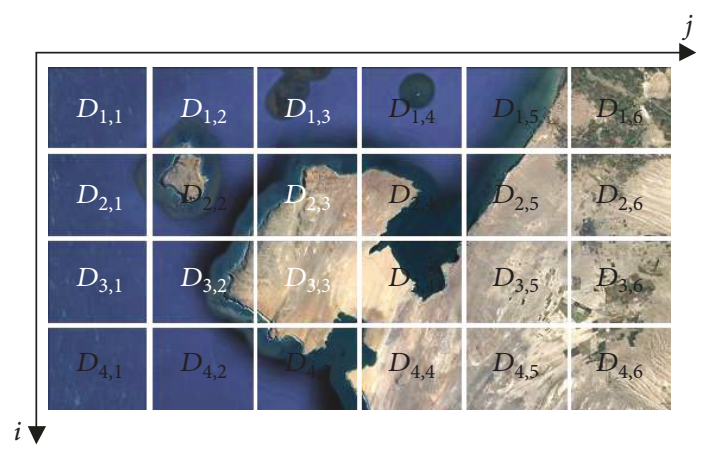

FIGURE 4: Illustration of the number of SURF features in each block.

to decrease. The diagram of local field attenuation effect is also demonstrated in Figure 4. It is supposed that block $D_{2,2}$ has been selected; then the normalized SURF feature amount $\widehat{S}_{i, j}$ of each local field with white color should be decayed. Assuming that the attenuation threshold is $T$, then $\widehat{S}_{i, j}$ is updated according to

$$
\widehat{S}_{i, j}=\widehat{S}_{i, j}-T \text {. }
$$

The entire diagram flow is presented in Figure 5.

The major steps of chessboard segmentation algorithm are as follows.

(1) Image Segmentation. In the first step, CSA splits the source image into $N \times M$ blocks. Simultaneously, the SURF features of each block will be extracted with the help of parallel computing technology.

(2) Data Normalization. Then the amount $S_{i, j}$ of SURF features of each block will be normalized to establish the weight matrix W.

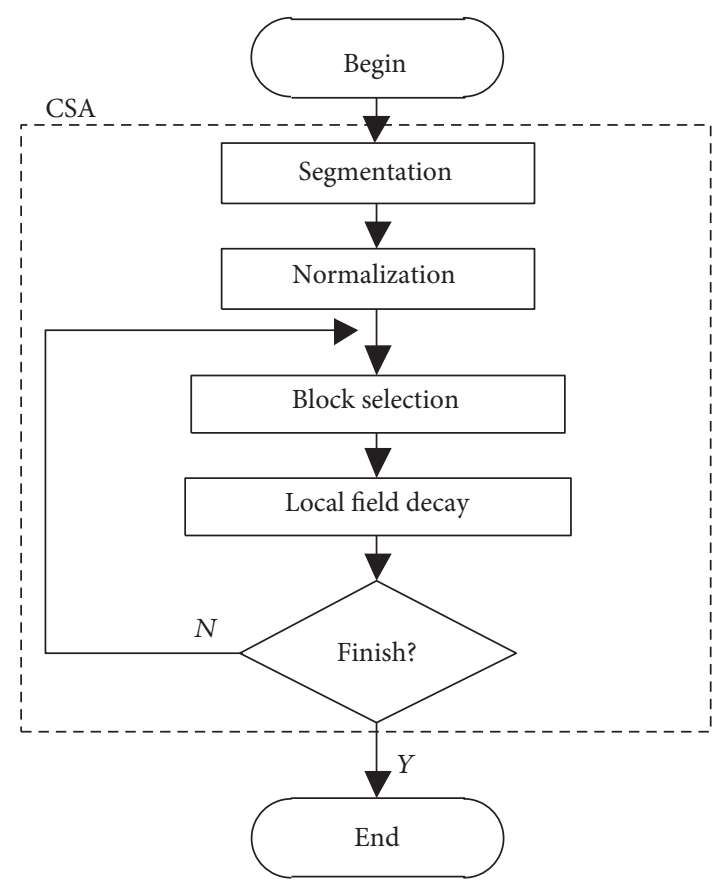

Figure 5: Diagram flow of CSA.

(3) Block Selection. In each step, the block with maximum weight among the candidate set will be selected and then be removed from the candidate set.

(4) Local Attenuation Effect. After the maximum weight has been selected, the weight coefficient around the selected block should minus a threshold $T$ to simulate the local attenuation effect.

Two parameters will affect the stability of CSA: the number of blocks in $x$ and $y$ directions. The experimental determination of the number of blocks that maximizes the stability of CSA is shown in Figure 6, which is based on an image registration task by using a collection of different natural landmarks. The terms $N_{x}$ and $N_{y}$ in this figure represent the number of blocks in $x$ direction and $y$ direction. Besides, the size of the object image that needs to be matched with the reference image is $800 \times 684$. The origin of an image coordinate system is located at the upper left corner of the corresponding image.

Figure 6 shows the repeatability for different combination of $N_{x}$ and $N_{y}$. The red marker is the best result corresponding to $100 \%$ repeatability. According to experimental results, the CSA works better when the following equation is satisfied:

$$
\frac{N_{y}}{N_{x}} \approx \frac{\text { image width }}{\text { image height }} .
$$

Besides, CSA threshold $T$ represents the extent of local attenuation effect and it is also an important parameter that will affect the CSA result. Here, a solution is proposed to 


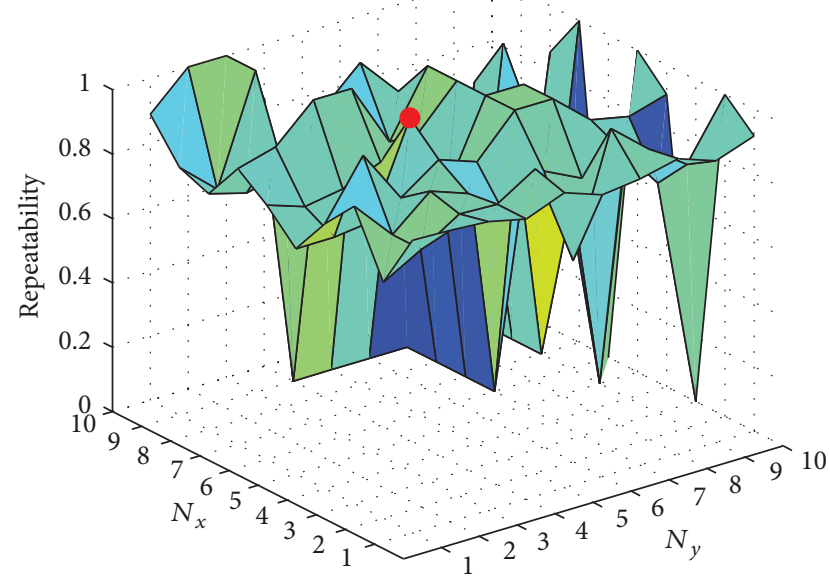

FIGURE 6: Repeatability for combined $N_{x}$ and $N_{y}$.

automatically calculate the threshold. A dataset is defined as follows:

$$
\alpha=\left\{w_{i, j}, i=1, \ldots, N, j=1, \ldots, M\right\},
$$

where $w_{i, j}$ is the weight coefficient in weight matrix. Then the threshold $T$ is equal to standard derivation of weight coefficients.

$$
T=\sqrt{\frac{1}{N \times M-1} \sum_{j}^{M} \sum_{i}^{N}\left(w_{i, j}-\bar{w}\right)^{2}},
$$

in which $\bar{w}$ is the mean of dataset $\alpha$. By this equation, a suitable threshold $T$ can be obtained.

Figures 7-9 present the results of CSA with different thresholds. In these figures, the selected blocks have been marked by green color. In Figure 7, the threshold $T=0$ means that the local attenuation effect is invalid and illustrates that these blocks whose feature number is not equal to 0 are selected. Figure 8 shows the CSA result with the threshold $T=0.1$ or 0.2 . And Figure 9 shows the CSA result with automatic determined threshold $T=0.3333$. It can be seen that Figure 9 gives the most typical and minimal number of blocks with the result of automatic threshold determination.

3.2. Random Sample Consensus Algorithm. Once the result of image registration is obtained, it is hard to analyze statistically the match ratio because it may have hundreds of matched key points in result. In this section, an algorithm called random sample consensus [12-14] is introduced to deal with the above problem. Besides, this algorithm also can be used to remove the wrong matched key points [15].

3.2.1. Perspective Transformation. Perspective transformation is a commonly used model to represent the relationship between two images from different views.

Figure 10 is a demonstration of perspective transformation. $P_{1}$ is a point in plane 1 , and its coordinate is $(x, y) . Q_{1}$

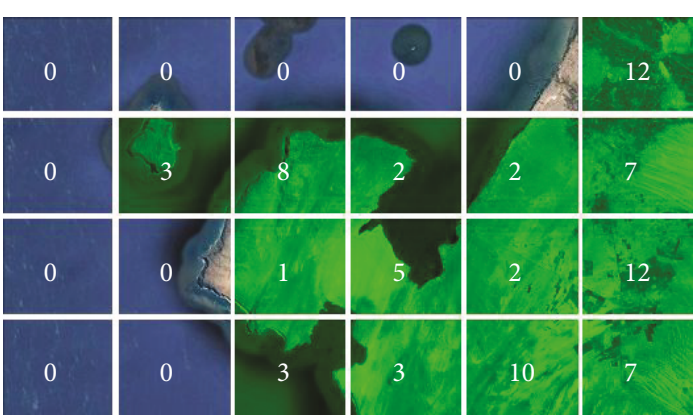

FIgURE 7: CSA result with threshold $T=0$.

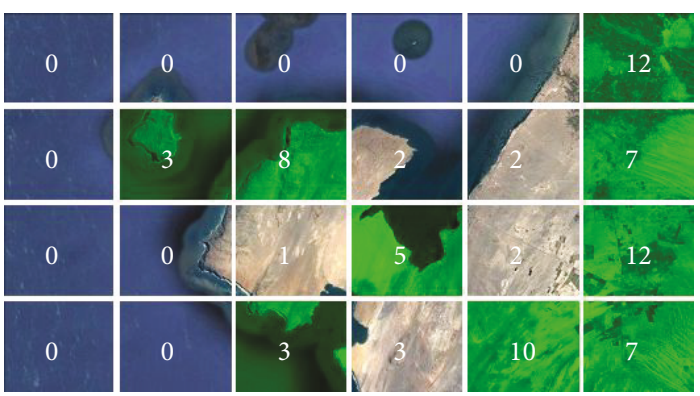

Figure 8: CSA result with threshold $T=0.1$ or 0.2 .

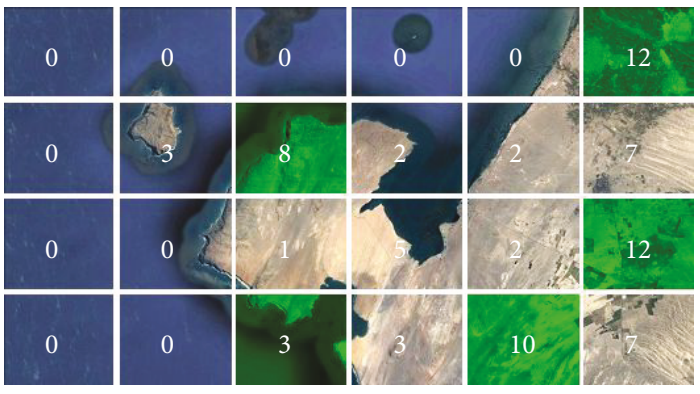

FIgURE 9: CSA result with automatic determined threshold $T=0.3333$.

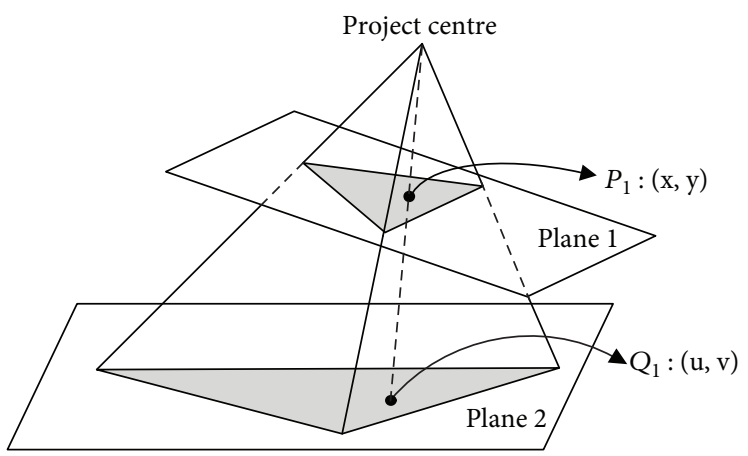

Figure 10: Perspective transformation. 
is the corresponding point in plane 2 with coordinate $(u, v)$. In order to get the relationship between $P_{1}$ and $Q_{1}$, a perspective transformation matrix $\mathbf{H}$ is defined as

$$
\mathbf{H}=\left[\begin{array}{ccc}
h_{1} & h_{2} & h_{3} \\
h_{4} & h_{5} & h_{6} \\
h_{7} & h_{8} & 1
\end{array}\right] .
$$

Then the relationship between $P_{1}$ and $Q_{1}$ is

$$
\lambda\left[\begin{array}{l}
u \\
v \\
1
\end{array}\right]=\mathbf{H}\left[\begin{array}{l}
x \\
y \\
1
\end{array}\right],
$$

where $\lambda$ is the scaling factor. By dividing the first and second rows with the third row, it will achieve

$$
\begin{aligned}
& u=\frac{h_{1} x+h_{2} y+h_{3}}{h_{7} x+h_{8} y+1}, \\
& v=\frac{h_{4} x+h_{5} y+h_{6}}{h_{7} x+h_{8} y+1} .
\end{aligned}
$$

Then we assume that $\widehat{\mathbf{H}}=\left[\begin{array}{lll}h_{1} & \ldots & h_{8}\end{array}\right]^{T}$; to transfer the above equation into the matrix form, we can obtain

$$
\left[\begin{array}{cccccccc}
x & y & 1 & 0 & 0 & 0 & -x u & -y u \\
0 & 0 & 0 & x & y & 1 & -x v & -y v
\end{array}\right] \widehat{\mathbf{H}}=\left[\begin{array}{l}
u \\
v
\end{array}\right] .
$$

In order to solve $\widehat{\mathbf{H}}$, four or more corresponding pairs are required. So, we suppose that there are four known corresponding groups $P_{1}, P_{2}, P_{3}, P_{4}$ and $Q_{1}, Q_{2}, Q_{3}, Q_{4}$, and satisfying

$$
\left[\begin{array}{cccccccc}
x_{1} & y_{1} & 1 & 0 & 0 & 0 & -x_{1} u_{1} & -y_{1} u_{1} \\
0 & 0 & 0 & x_{1} & y_{1} & 1 & -x_{1} v_{1} & -y_{1} v_{1} \\
& & & & \ldots & & & \\
x_{4} & y_{4} & 1 & 0 & 0 & 0 & -x_{4} u_{4} & -y_{4} u_{4} \\
0 & 0 & 0 & x_{4} & y_{4} & 1 & -x_{4} v_{4} & -y_{4} v_{4}
\end{array}\right]\left[\begin{array}{c}
h_{1} \\
h_{2} \\
\vdots \\
h_{7} \\
h_{8}
\end{array}\right]=\left[\begin{array}{c}
u_{1} \\
v_{1} \\
\vdots \\
u_{4} \\
v_{4}
\end{array}\right],
$$

it can be simplified as

$$
\mathbf{A} \widehat{\mathbf{H}}=\mathbf{b} .
$$

Because of distortion, noise, or other reasons, the above equation may be a contradictory equation. Therefore, the least squares method is applied to find the satisfied result

$$
\widehat{\mathbf{H}}=\left(\mathbf{A}^{T} \mathbf{A}\right)^{-1} \mathbf{A}^{T} \mathbf{b} .
$$

Eventually, the perspective transformation matrix $\mathbf{H}$ can be recovered from $\widehat{\mathbf{H}}$.

Assuming that $P(x, y)$ is the key point in the first picture. $Q(u, v)$ is the corresponding matched key point in the second image. $\mathbf{H}_{1}$ is the perspective transformation matrix between the first and second image. Then we have

$$
\lambda\left[\begin{array}{lll}
x^{\prime} & y^{\prime} & 1
\end{array}\right]^{T}=\mathbf{H}_{1}\left[\begin{array}{lll}
x & y & 1
\end{array}\right]^{T},
$$

where $\left(x^{\prime}, y^{\prime}\right)$ is the corresponding point after perspective projection transformation on key point $P$. Then the perspective projection error can be defined as follows:

$$
\operatorname{Err}=\sqrt{\left(x^{\prime}-u\right)^{2}+\left(y^{\prime}-v\right)^{2}} .
$$

3.2.2. RANSAC Algorithm Progress. Figure 11 shows the diagram flow of RANSAC algorithm. The main stages of RANSAC algorithm are described as follows:

(1) Initialization. The threshold of minimum perspective project error $T_{\text {ppe }}$ should be initialized at first. Besides, the maximum iterator time $M_{\text {iter }}$ should also be initialized here.

(2) Perspective Transformation Solving. The perspective transformation $\mathbf{H}$ can be solved by least squares method with four groups of key points, among which four key points are randomly selected from the dataset of source image key points. The other four key points are randomly selected from the dataset of target image key points.

(3) Perspective Matrix Validation. Once a new perspective projection error has been obtained in each iterator, a validation needs to be performed. The minimum of perspective projection error Err between the key points of the source image and the key points of the target image will be calculated. If Err is smaller than the smallest perspective projection error $E_{\min }$ which is stored before, the minimum perspective projection error $E_{\min }$ will be updated.

(4) Loop Termination. If $E_{\min }>T_{\text {ppe }}$ and iterator $>M_{\text {iter }}$, the loop will go back to step 2; otherwise, the loop will be terminated.

\section{Time Consumption Comparison}

In order to verify the advantage of the proposed CSA-SURF algorithm, the SURF and PCA-SURF algorithms are used for comparison. Feature extraction and matching progress are different for the above three algorithms, of which the time complexities are discussed in the following.

4.1. Time Consumption for Feature Extraction. It is well known that SIFT or SURF algorithms use the sliding window method to detect local extrema. PCA-SURF and CSA-SURF, having the same mechanism, are the improved algorithms based on SURF. However, there are some differences among them. In order to descript more clearly, the width and height of the image are defined as $I_{\mathrm{w}}$ and $I_{\mathrm{h}}$ and the step size is $\sigma$. The time for solving the eigenvalue and eigenvector is $T_{\text {eig. }}$. The time for transferring the old feature space to the new feature space is $T_{\text {tran }}$. The time for computing the local 


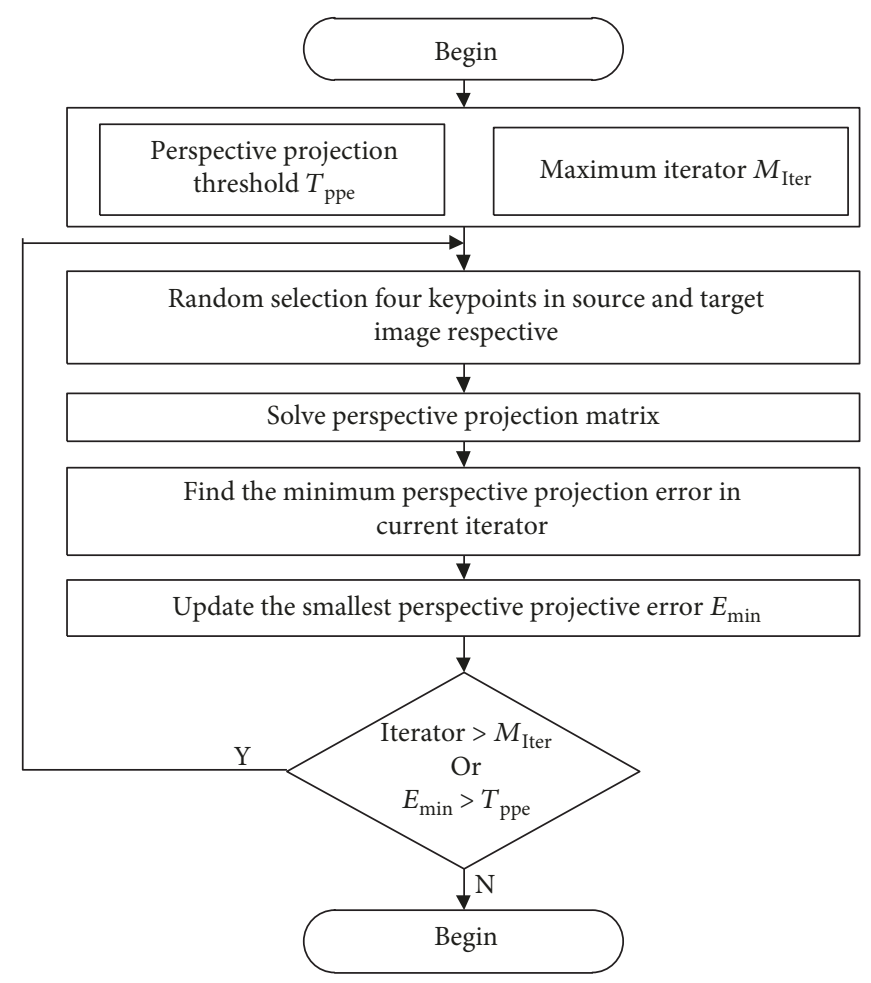

Figure 11: Diagram flow of RANSAC algorithm.

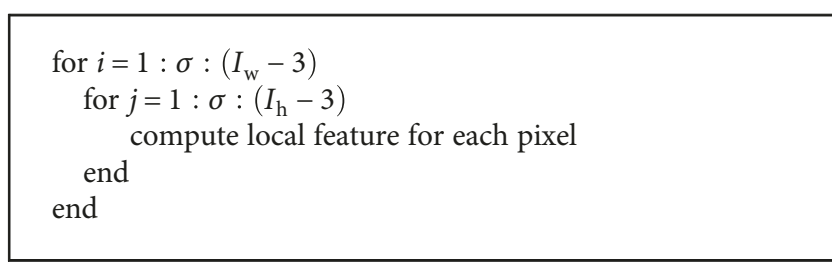

PSEUdocode 1: Pseudocode for SURF feature extraction progress.

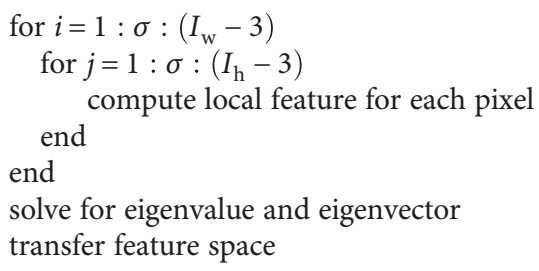

PsEudocode 2: Pseudocode for PCA-SURF feature extraction progress.

feature is $T_{\text {local }}$. The extraction times of SURF, PCA-SURF, and CSA-SURF are $T_{\text {surf }}^{e}, T_{\mathrm{pca} \text {-surf }}^{e}$, and $T_{\text {csa-surf }}^{e}$, respectively. The pseudocodes for the feature extraction progress of SURF, PCA-SURF, and CSA-SURF algorithms are presented as in Pseudocodes 1, 2, and 3.

The extraction time of SURF, PCA-SURF, and CSA-SURF can be calculated by

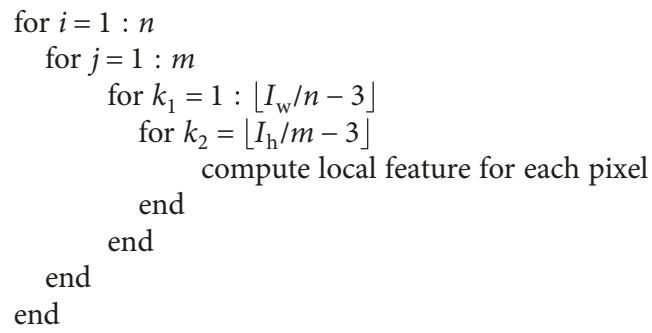

Pseudocode 3: Pseudocode for CSA-SURF feature extraction progress.

$$
\begin{aligned}
T_{\text {surf }}^{e} & =\left\lfloor\frac{\left(I_{\mathrm{w}}-3\right)}{\sigma}\right\rfloor\left\lfloor\frac{\left(I_{\mathrm{h}}-3\right)}{\sigma}\right\rfloor T_{\text {local }}, \\
T_{\text {pca-surf }}^{e} & =\left\lfloor\frac{\left(I_{\mathrm{w}}-3\right)}{\sigma}\right\rfloor\left\lfloor\frac{\left(I_{\mathrm{h}}-3\right)}{\sigma}\right\rfloor T_{\text {local }}+T_{\text {eig }}+T_{\text {tran }}, \\
T_{\text {csa-surf }}^{e} & =n m\left\lfloor\frac{I_{\mathrm{w}} / n-3}{\sigma}\right\rfloor\left\lfloor\frac{I_{\mathrm{h}} / m-3}{\sigma}\right\rfloor T_{\text {local }},
\end{aligned}
$$

satisfying

$$
T_{\text {csa-surf }}^{e}<T_{\text {surf }}^{e}<T_{\text {pca-surf }}^{e}
$$

in which the symbol $\lfloor\cdot\rfloor$ is a rounding down function.

The relationship of (24) can be proven as follows.

Obviously, we have 


$$
\begin{aligned}
n m\left\lfloor\frac{\left(I_{\mathrm{w}} / n-3\right)}{\sigma}\right\rfloor\left\lfloor\frac{\left(I_{\mathrm{h}} / m-3\right)}{\sigma}\right\rfloor & <\frac{n m\left(I_{\mathrm{w}} / n-3\right)\left(I_{\mathrm{h}} / m-3\right)}{\sigma^{2}}, \\
n m\left\lfloor\frac{\left(I_{\mathrm{w}} / n-3\right)\left(I_{\mathrm{h}} / m-3\right)}{\sigma^{2}}\right\rfloor & =\frac{\left(I_{\mathrm{w}}-3 n\right)\left(I_{\mathrm{h}}-3 m\right)}{\sigma^{2}}, \\
\frac{\left(I_{\mathrm{w}}-3 n\right)\left(I_{\mathrm{h}}-3 m\right)}{\sigma^{2}} & <\left\lfloor\frac{I_{\mathrm{w}}-3}{\sigma}\right\rfloor\left\lfloor\frac{I_{\mathrm{h}}-3}{\sigma}\right\rfloor .
\end{aligned}
$$

Combining (26) and (27), we obtain

$$
n m\left\lfloor\frac{\left(I_{\mathrm{w}} / n-3\right)}{\sigma}\right\rfloor\left\lfloor\frac{\left(I_{\mathrm{h}} / m-3\right)}{\sigma}\right\rfloor<\left\lfloor\frac{\left(I_{\mathrm{w}}-3\right)}{\sigma}\right\rfloor\left\lfloor\frac{\left(I_{\mathrm{h}}-3\right)}{\sigma}\right\rfloor,
$$

that is,

$$
T_{\text {csa-surf }}^{e}<T_{\text {surf }}^{e}
$$

Obviously,

$$
T_{\text {surf }}^{e}<T_{\text {pca-surf }}^{e}
$$

Therefore, (24) has been verified.

4.2. Time Consumption of Matching. In CSA-SURF, the image is divided into $n$ blocks in horizontal and $m$ blocks in vertical. The number of extracted features for SURF and PCA-SURF is $N$, while the corresponding number for CSASURF is $M$ and is much smaller than $N$. Besides, the dimension of PCA-SURF is $l$. The time consumption for two features matching is $T_{\text {match }}$. The matching time for SURF, PCA-SURF, and CSA-SURF are $T_{\text {surf }}^{m}, T_{\text {pca-surf }}^{m}$, and $T_{\text {csa-surf }}^{m}$ and can be calculated by

$$
\begin{aligned}
T_{\text {surf }}^{m} & =N^{2} T_{\text {match }}, \\
T_{\text {pca-surf }}^{m} & =\frac{N^{2} l}{64} T_{\text {match }}, \\
T_{\text {csa-surf }}^{m} & =M^{2} T_{\text {match }} .
\end{aligned}
$$

As $M$ is much less than $N$, then we have

$$
T_{\text {csa-surf }}^{m}<T_{\text {pca-surf }}^{m}, \quad \text { when } M \ll N \text {, }
$$

which can be proven as follows.

As we can see,

$$
\begin{aligned}
M^{2} & <N^{2}, \\
\frac{N^{2} l}{64} & <N^{2} .
\end{aligned}
$$

Therefore, we have

$$
\begin{aligned}
& T_{\text {pca-surf }}^{m}<T_{\text {surf }}^{m}, \\
& T_{\text {csa-surf }}^{m}<T_{\text {surf }}^{m} .
\end{aligned}
$$

If $M \ll N$, then

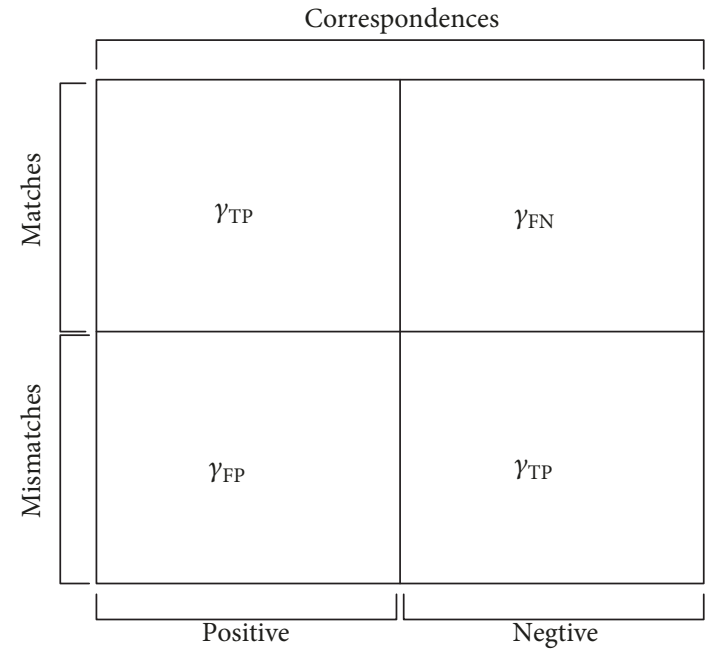

FIGURE 12: Relationship of different symbols.

$$
\left(\frac{M}{N}\right)^{2} \longrightarrow \text { small number. }
$$

For the spacecraft autonomous navigation problem using natural landmarks, only 5 to 10 features satisfying certain relative distance constraints are required; therefore, it is easy to guarantee that $(M / N)^{2}<N^{2} / l$ and then we have $T_{\text {csa-surf }}^{m}<T_{\text {pca-surf }}^{m}$.

From the above comparison, the proposed CSA-SURF method consumes the least time.

\section{Evaluation}

5.1. Evaluation Metrics. In order to descript the repeatability performance, the following evaluation metrics are defined in this section. Positive in this paper means the key points that can be matched. Negative means the key points that cannot be matched.

Then we suppose that $\gamma_{\mathrm{TP}}$ is the number of positive key points that are correct matches. $\gamma_{\mathrm{FP}}$ is the number of negative key points that are false matches. $\gamma_{\mathrm{TN}}$ is the number of negative key points that correct mismatches. And $\gamma_{\mathrm{FN}}$ is the number of negative key points that are false mismatches. To explain it more clearly, Figure 12 shows the relationship of different symbols.

Then we have the following definitions:

$$
\begin{aligned}
\text { Precision } & =\frac{\gamma_{\mathrm{TP}}}{\gamma_{\mathrm{TP}}+\gamma_{\mathrm{FP}}}=\frac{\text { correct matches }}{\text { positive }}, \\
\text { Recall } & =\frac{\gamma_{\mathrm{TP}}}{\gamma_{\mathrm{TP}}+\gamma_{\mathrm{FN}}}=\frac{\text { correct matches }}{\text { positive }},
\end{aligned}
$$

Precision means the proportion of correct matches to positive. Recall means the proportion of correct matches to matches. Recall and precision are conflicting, so a recal 

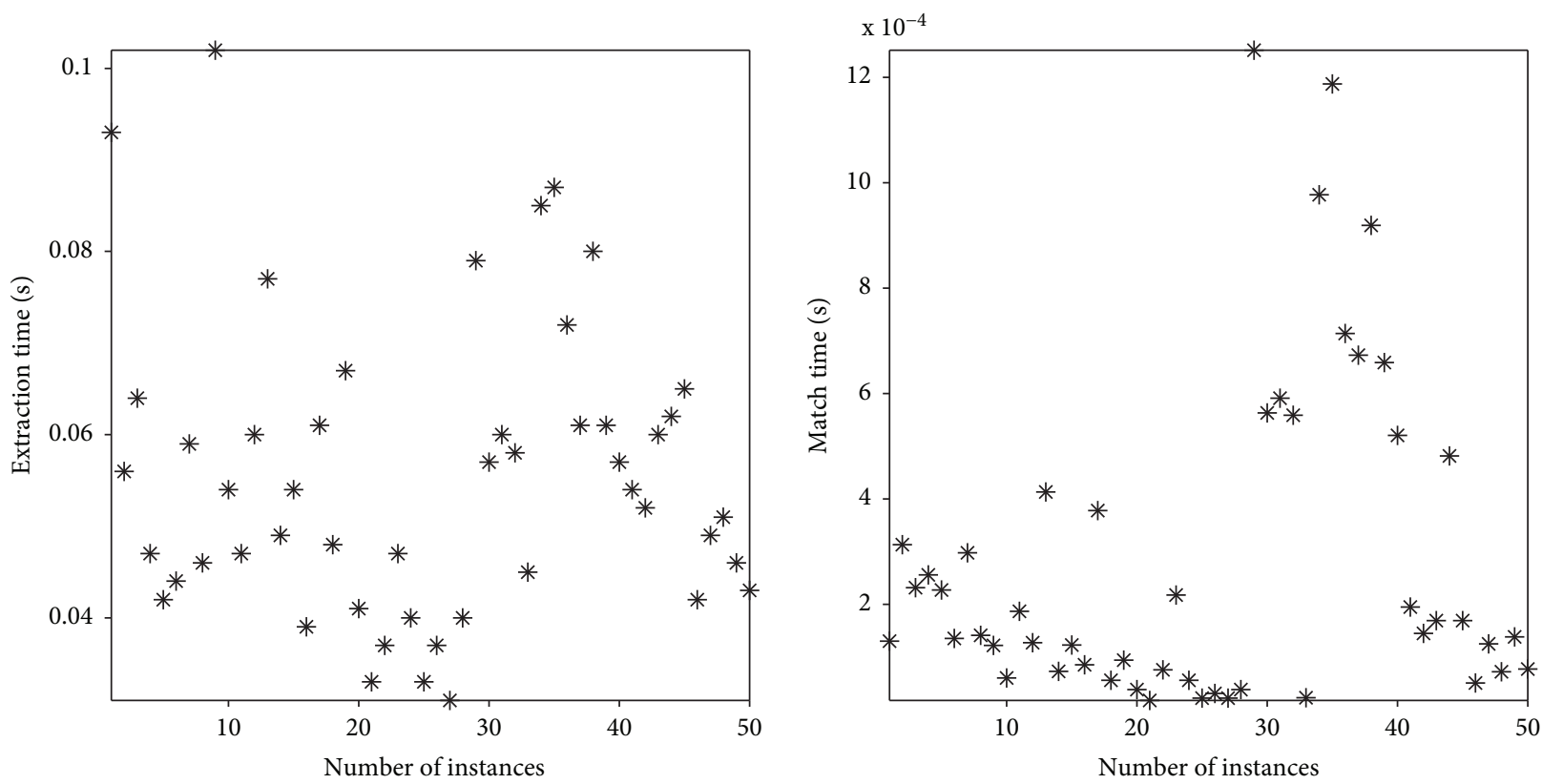

FIgURE 13: Time consumption for SURF.
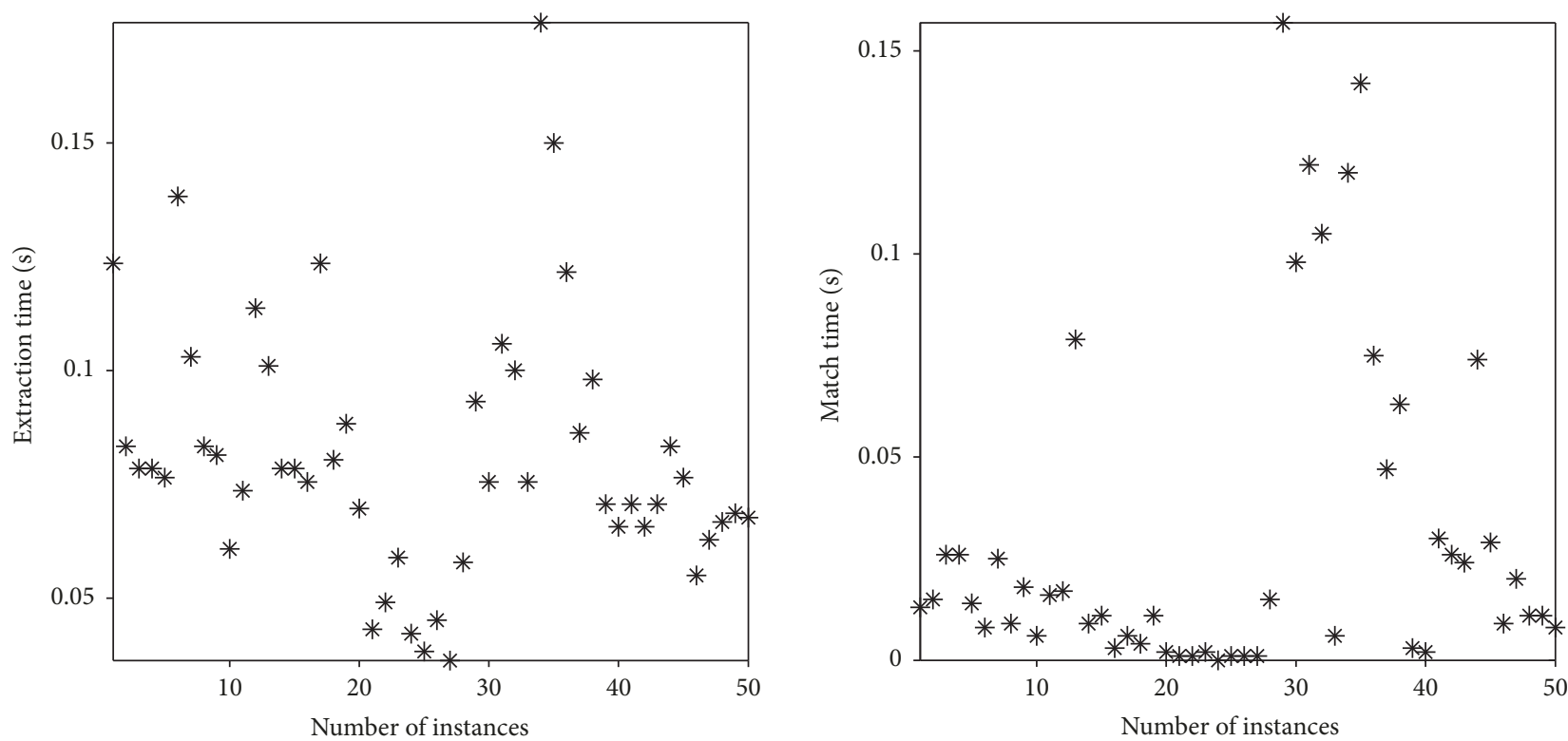

FIgURE 14: Time consumption for PCA-SURF.

1 versus precision graph will help in analyzing the repeatability performance.

5.2. Speed Test. The following experiments are tested on Core I5 $2.3 \mathrm{GHz}$ CPU, 6GB RAM laptop with Windows 7 operation system. VS2013 and OpenCV2.4 are used to carry out all the experiments. And all datasets are from an open source database called UC Merced Land Use Dataset. And the resolution is $256 \times 256$.

Figures 13-15 are the matching comparison results of fifty images about SURF, PCA-SURF, and CSA-SURF. It can be seen that PCA-SURF consumes much more time than SURF and CSA-SURF during feature extraction process. The CSA-SURF algorithm works faster than SURF and PCA-SURF algorithms for both feature extraction and matching processes.

5.3. Repeatability Test. Figure 16 shows the repeatability of different methods for different conditions. But recall and precision may be conflict in some condition, so a recall versus precision graph has been established to help us analyze the repeatability performance. The red line is the test under 

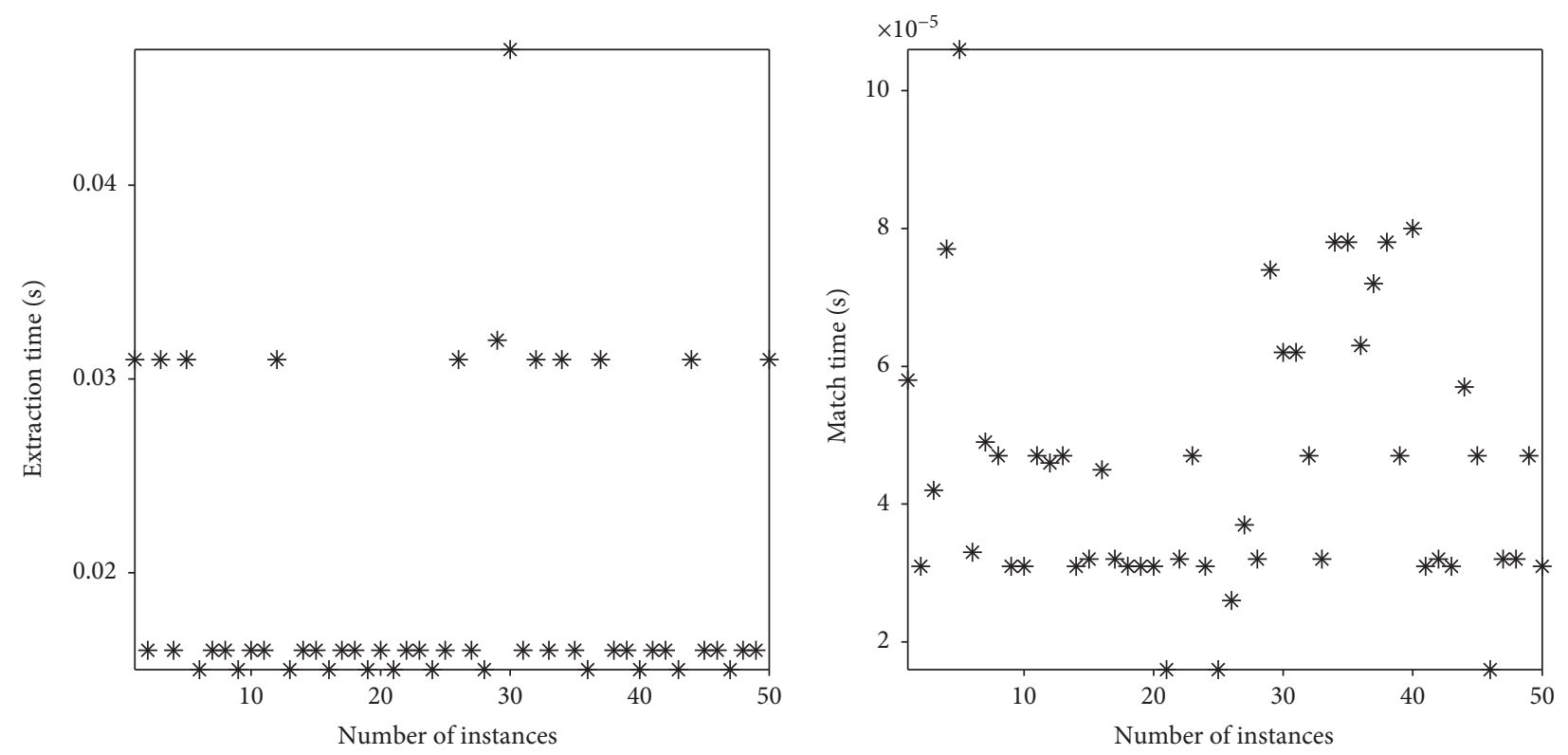

FIGURE 15: Time consumption for CSA-SURF.

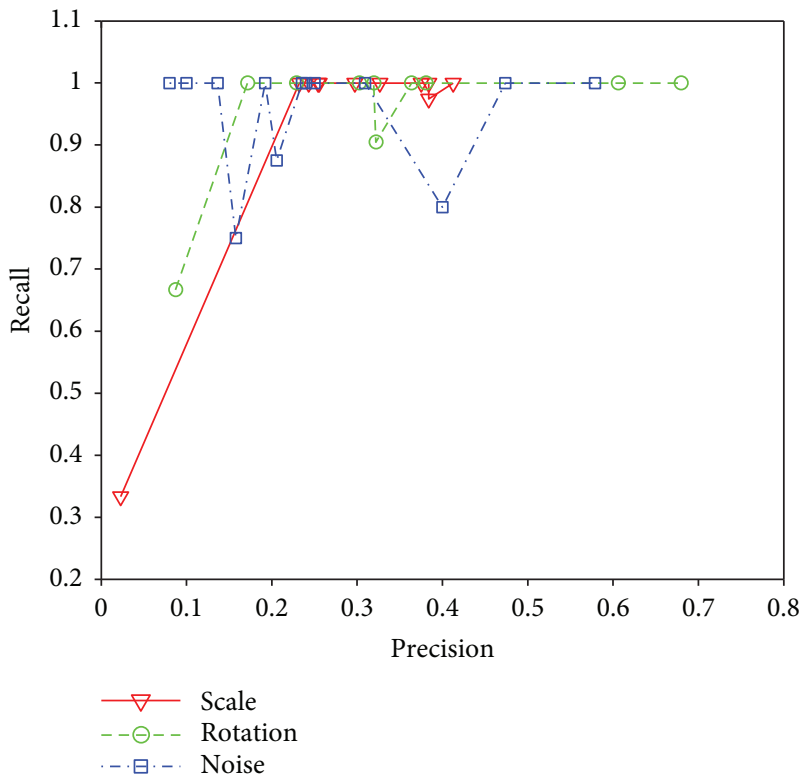

FIGURE 16: Recall versus precision graph of three methods under different conditions.

scaling; it can be seen that CSA-SURF performs worse when precision $<0.2$ and its performance is not stable when $0.2<$ precision $<0.45$. For precision $<0.45$, it works well with recall $=1$. The green line represents the test of CSA-SURF algorithm under rotation. Its performance is much the same as the solid line's case, while its unstable region is $0.2<$ precision $<0.4$. The blue line represents the test of CSA-SURF algorithm under salt and pepper noise [16] with a noise density equal to 0.35 . It can be seen that CSA-SURF algorithm is not stable under salt and pepper noise, but recall still maintains its value above 0.7 .

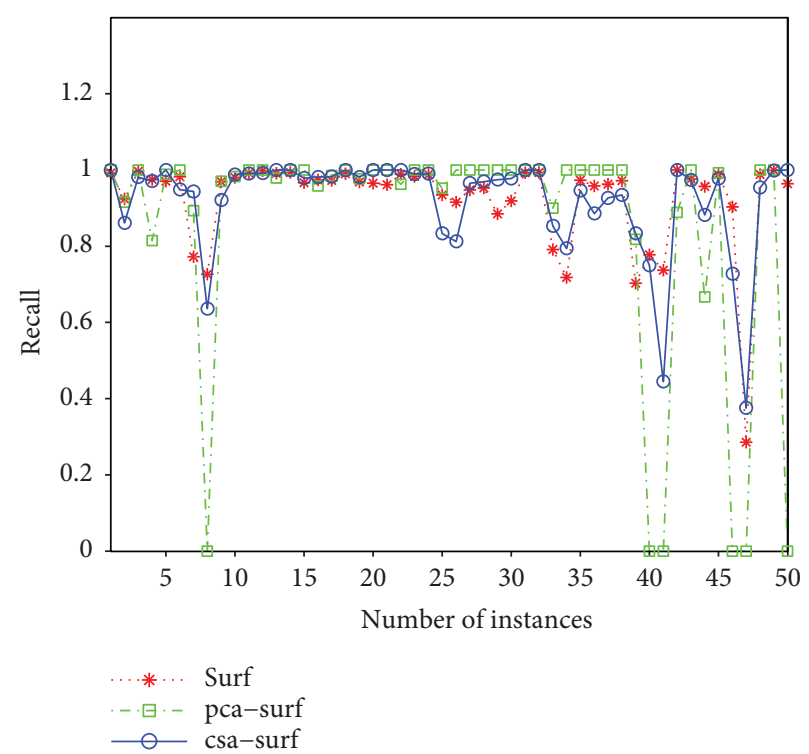

Figure 17: Recall of different methods.

Furthermore, the above dataset with fifty images is also used to test the repeatability performance of the three methods. Figure 17 is the recall of different methods for these images. The results show that the repeatability of SURF and CSA-SURF is very close. Therefore, CSA-SURF can speed up the registration progress without losing its repeatability performance. However, PCA-SURF is unstable as it can get the correct matches in some conditions.

5.4. Application on Natural Landmark Registration. The major purpose of this paper is to apply the proposed method into natural landmark registration task. Five different kinds of natural landmarks, including island, airport, 

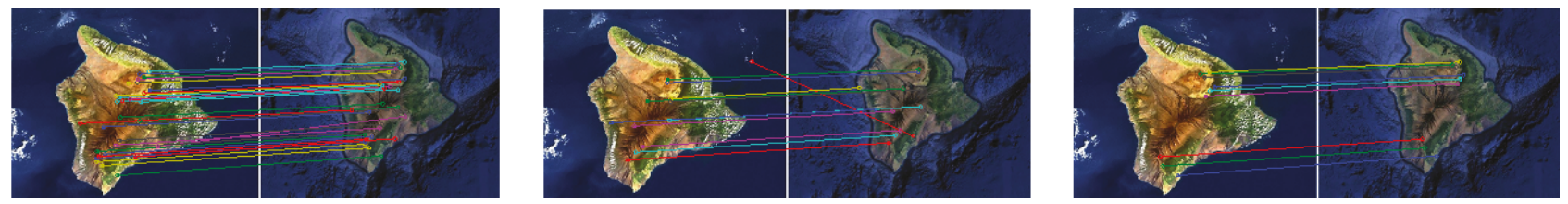

FIGURE 18: Hawaiian island registration result with SURF, PCA-SURF, and CSA-SURF.
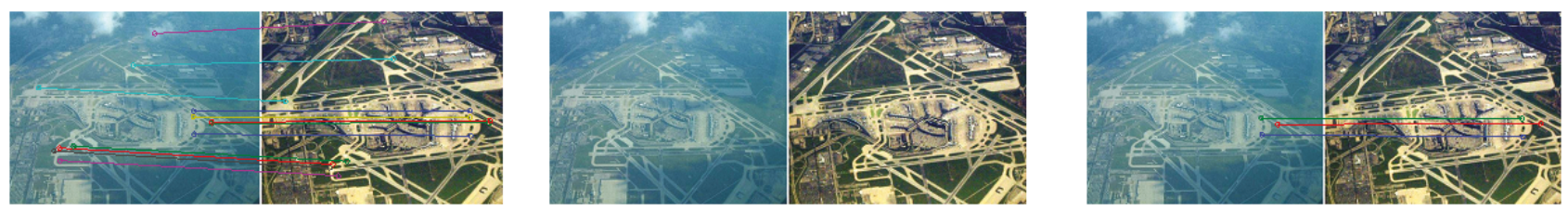

Figure 19: O'Hare International Airport registration result with SURF, PCA-SURF, and CSA-SURF.
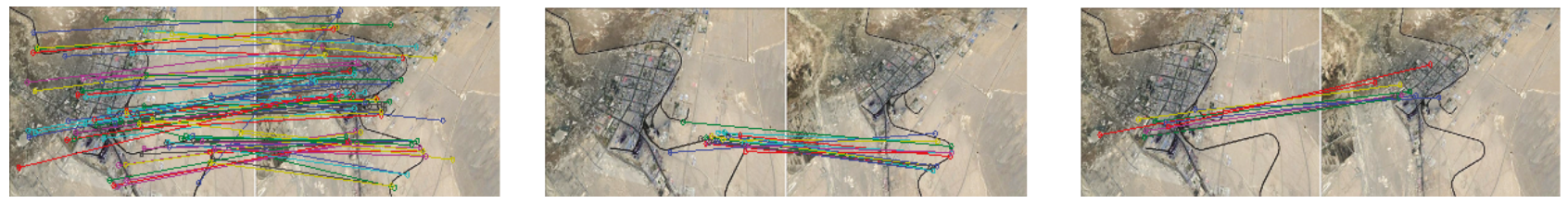

FIGURE 20: Qingzang railway registration result with SURF, PCA-SURF, and CSA-SURF.
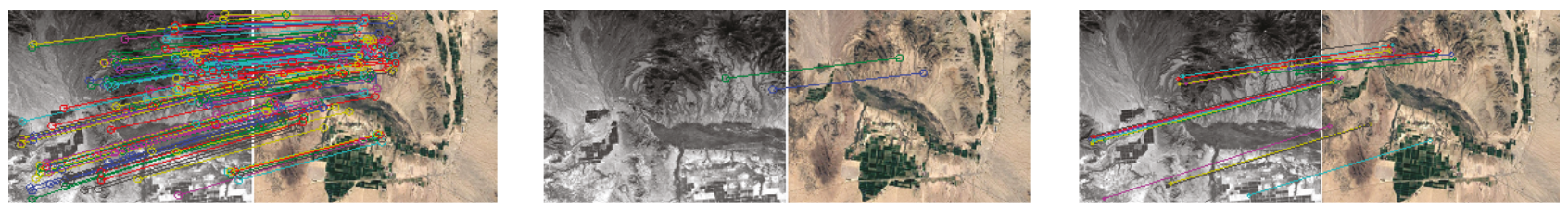

FIGURE 21: River landmark registration result with SURF, PCA-SURF, and CSA-SURF.
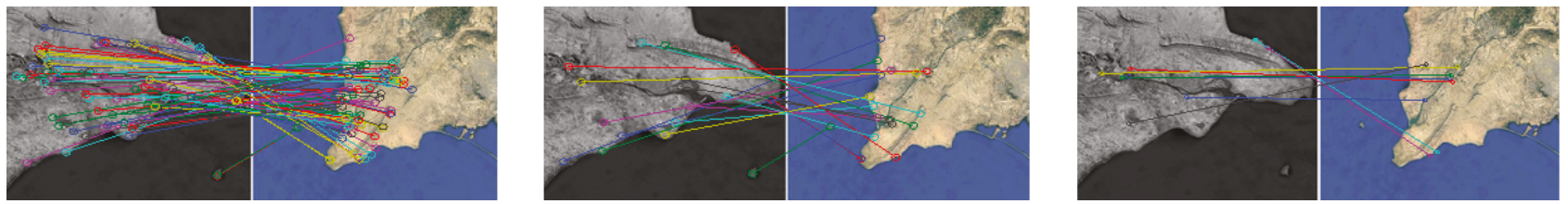

FIGURE 22: Coastline landmark registration result with SURF, PCA-SURF, and CSA-SURF.

railway, river, and coastline, are collected for the demonstration testing.

Figure 18 presents the registration results for Hawaiian islands with different algorithms, in which SURF and CSASURF work fine but PCA-SURF has a wrong match. Figure 19 shows the matching results of two O'Hare International Airport images with different algorithms, indicating that the PCA-SURF algorithm is unstable as it cannot find any matched key points in this example. Figures $20-22$ are the matching results of Qingzang railway, river, and coastline images with different algorithms.

It can be seen from the above testing results that CSA-SURF algorithm works well for different images with significant contour features. The proposed CSA-SURF algorithm, which is improved from SURF, keeps the repeatability of SURF unchanged and improves the image matching speed. Many other pictures have also been tested; however, the results are not presented for the page limitation of the paper.

\section{Conclusion}

Because of the restricted computational resource and horrible environment for an autonomous navigation system, traditional image registration methods cannot satisfy practical requirements in speed. A novel algorithm called chessboard 
segmentation algorithm is proposed to solve the above problem. Because the new method is based on SURF features, it inherits lots of their advantages, such as scale and rotation invariant properties. To verify the improvement of the proposed algorithm, the PCA-SURF algorithm which was proposed in recent years is also presented in this paper for comparison. Besides, RANSAC algorithm is applied to remove the false negative key points to further improve the accuracy of the proposed algorithm. Thorough experiments have been carried out to demonstrate the performance of the proposed method; the corresponding simulation results show great improvement in image registration speed without losing repeatability. Finally, the CSASURF algorithm is applied to natural landmark registration task, showing that it works fine. The proposed method is a good candidate for image registration task for spacecraft autonomous navigation based on natural landmarks.

\section{Conflicts of Interest}

The authors declare that they have no conflicts of interest.

\section{Acknowledgments}

The authors would like to express their acknowledgment for the support provided by the National Natural Science Foundation of China (nos. 61403197 and 61673212), the Natural Science Foundation of Jiangsu Province (no. BK20140830), the National Key Research and Development Plan (no. 2016YFB0500901), and the Open Fund of National Defense Key Discipline Laboratory of Micro-Spacecraft Technology (no. HIT.KLOF.MST.201705).

\section{References}

[1] A. C. Vigneron, A. H. J. de Ruiter, B. V. Burlton, and W. K. H. Soh, "Nonlinear filtering for autonomous navigation of spacecraft in highly elliptical orbit," Acta Astronautica, vol. 126, pp. 138-149, 2016.

[2] S. Sha, C. Jianer, and L. Sanding, "A fast matching algorithm based on K-degree template," in 2009 4th International Conference on Computer Science \& Education, pp. 1967-1971, Nanning, China, 2009.

[3] Z. Xu, Y. Liu, S. Du, P. Wu, and J. Li, "DFOB: detecting and describing features by octagon filter bank for fast image matching," Signal Processing: Image Communication, vol. 41, pp. 61-71, 2016.

[4] P. Zhao, Z. Bai, and W. Fan, "Research of fast image matching based on PCA," Computer Technology and Its Applications, vol. 4, pp. 132-134, 2010.

[5] D. He and P. Jiang, "Fast image matching algorithm based on discrete Hartley transform," Modern Defense Technology, vol. 44, no. 5, pp. 61-65, 2016.

[6] Y. Ke and R. Sukthankar, "PCA-SIFT: a more distinctive representation for local image descriptors," in Proceedings of the 2004 IEEE Computer Society Conference on Computer Vision and Pattern Recognition, 2004. CVPR 2004, pp. II-506II-513, Washington, DC, USA, 2004.

[7] J. Yang, D. Zhang, A. F. Frangi, and J.-y. Yang, "Two-dimensional PCA: a new approach to appearance-based face representation and recognition," IEEE Transactions on Pattern Analysis and Machine Intelligence, vol. 26, no. 1, pp. 131-137, 2004.

[8] H. Bay, A. Ess, T. Tuytelaars, and L. Van Gool, "Speeded-up robust features (SURF)," Computer Vision and Image Understanding, vol. 110, no. 3, pp. 346-359, 2008.

[9] M. Bleyer and M. Gelautz, "Graph-cut-based stereo matching using image segmentation with symmetrical treatment of occlusions," Signal Processing: Image Communication, vol. 22, no. 2, pp. 127-143, 2007.

[10] A. Pancham, D. Withey, and G. Bright, "Tracking image features with PCA-SURF descriptors," in 14th IAPR International Conference on Machine Vision Applications (MVA), pp. 365-368, Tokyo, Japan, 2015.

[11] E. E. Maraş, M. Caniberk, and H. H. Maraş, "Automatic coastline detection using image enhancement and segmentation algorithms," Polish Journal of Environmental Studies, vol. 25, no. 6, pp. 2519-2525, 2016.

[12] Y. Wang, J. Zheng, Q. Z. Xu, B. Li, and H. M. Hu, "An improved RANSAC based on the scale variation homogeneity," Journal of Visual Communication and Image Representation, vol. 40, pp. 751-764, 2016.

[13] Y. Chen, Q. Sun, H. Xu, and L. Geng, "Matching method of remote sensing images based on SURF algorithm and RANSAC algorithm," Jisuanji Kexue yu Tansuo, vol. 6, no. 9, pp. 822-828, 2012.

[14] Y. Zhao, R. Hong, and J. Jiang, "Visual summarization of image collections by fast RANSAC," Neurocomputing, vol. 172, pp. 48-52, 2016.

[15] F. Yang, J. Guo, and J. Wang, "Image mismatching eliminating algorithm using structural similarity and geometric constraint," Journal of Signal Processing, vol. 32, no. 1, pp. 83-90, 2016.

[16] R. H. Chan, Chung-Wa, and M. Nikolova, "Salt-and-pepper noise removal by median-type noise detectors and detailpreserving regularization," IEEE Transactions on Image Processing, vol. 14, no. 10, pp. 1479-1485, 2005. 


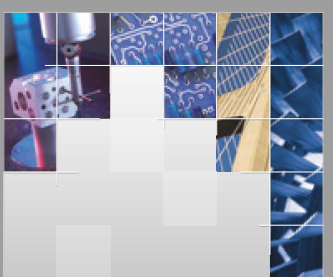

\section{Enfincering}
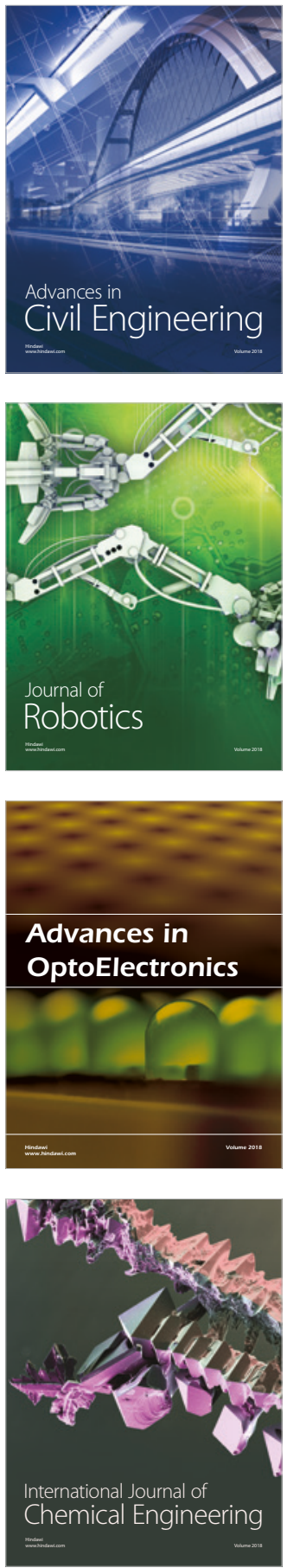

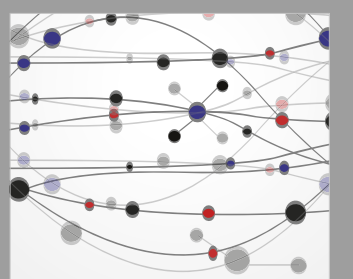

\section{Rotating \\ Machinery}

The Scientific World Journal

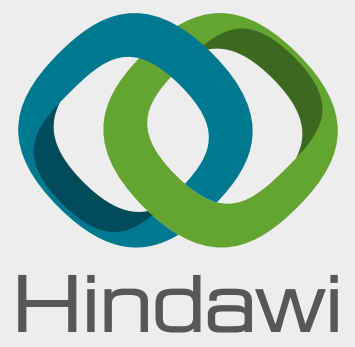

Submit your manuscripts at

www.hindawi.com
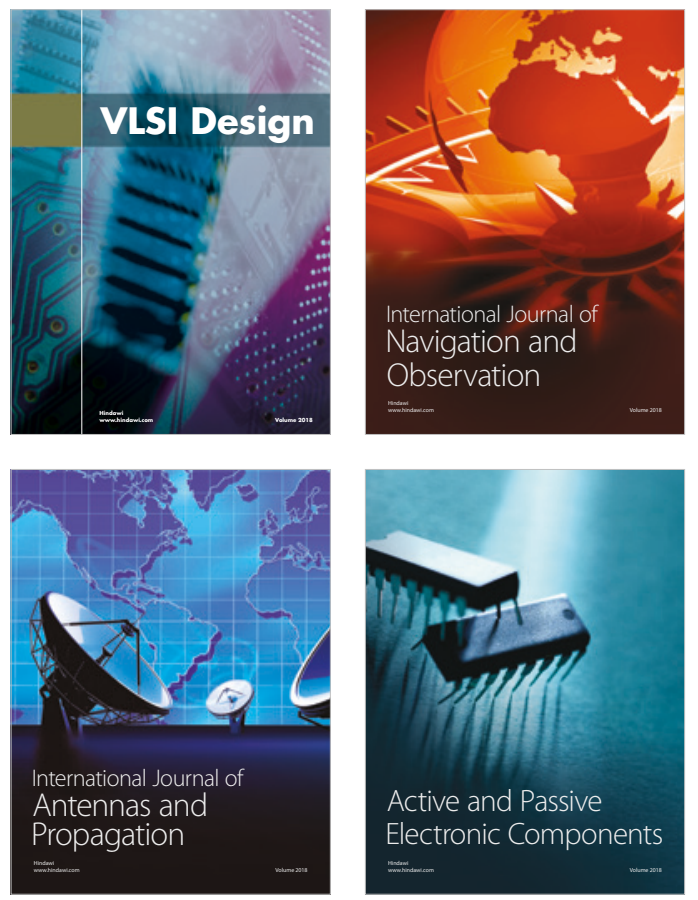
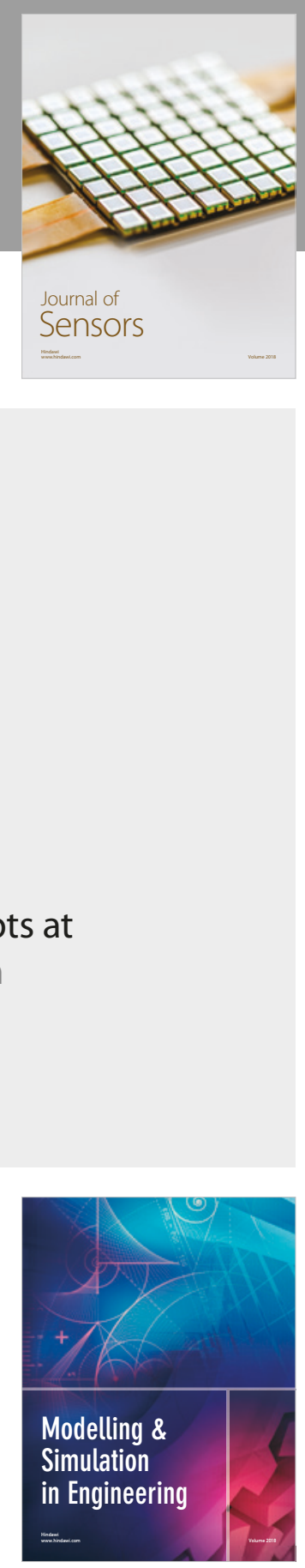

\section{Advances \\ Multimedia}
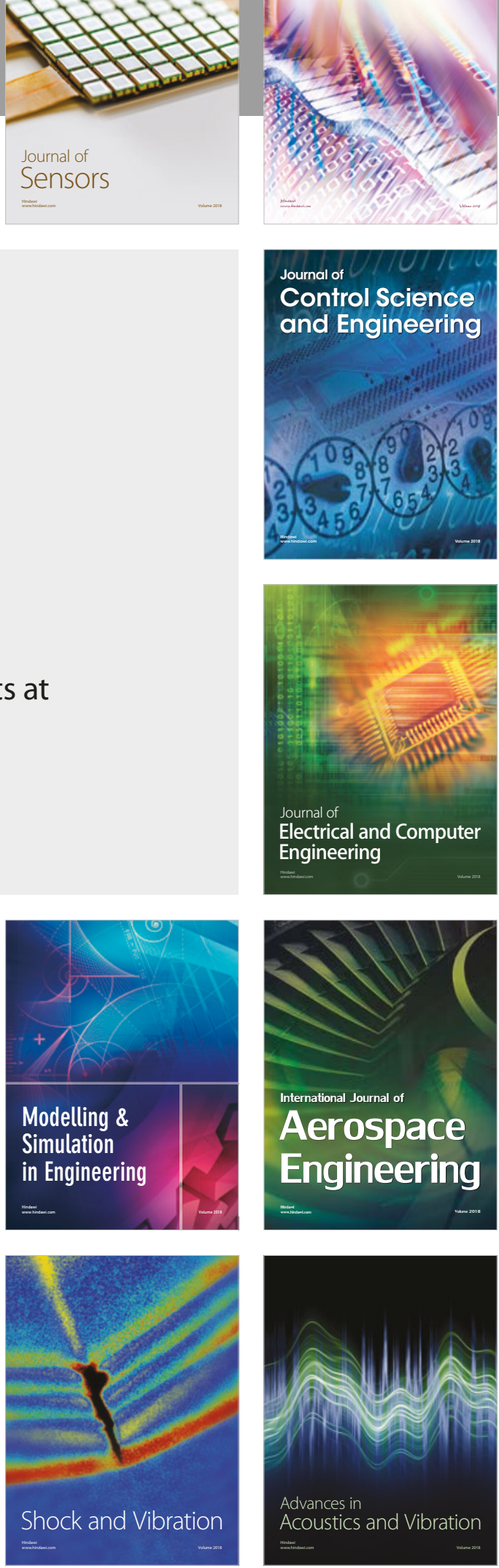